\title{
Lung metastasectomy for colorectal cancer: The impression of benefit from uncontrolled studies was not supported in a randomized controlled trial
}

Tom Treasure, MD, MS, FRCS, FRCP, ${ }^{a}$ Joel Dunning, PhD, FRCS(CTh), ${ }^{b}$

Norman R. Williams, BSc, PhD, CSci, MICR, FIBMS, ${ }^{\mathrm{c}}$ and Fergus Macbeth, MA, DM, FRCP, FRCR, MBA ${ }^{\mathrm{d}}$

The Society of Thoracic Surgeons expert consensus document on pulmonary metastasectomy states:

Since 1980, greater than 1,000 publications addressed PM [pulmonary metastasectomy], without a single randomized controlled trial. Most of the studies are surgical series, usually from a single institution, and include single or multiple pathologies. The pool of patients from which metastasectomy patients derive is not reported, allowing no comparative survival analysis. Historical controls are used or metastatic disease survival is assumed to be zero, a contention not supported by the literature.

The Society of Thoracic Surgeons statement confirms the widely held assumption of zero survival, as the basis of a practice, unsupported by adequate evidence. No randomized controlled trial (RCT) was found. We have recently published the Pulmonary Metastasectomy in Colorectal Cancer (PulMiCC) RCT, which puts something into that void ${ }^{1}$ (Figure 1).

A search in the late 2000s for evidence about colorectal cancer (CRC) lung metastasectomy, returned 101 publications reporting practice from the $1960 \mathrm{~s}^{2}$. Most offered no estimate of what survival might have been without surgery. Only 1 article attempted to address the question. Forty years ago, under the title "The effect of metastasectomy: Fact or fiction?" Åberg and colleagues ${ }^{3}$ wrote, "It has been assumed, implied, or claimed that the 5-year survival without operation is nil. Control material is, however, lacking." They had survival results for 70 patients who had lung metastasectomy between 1961 and 1978 (Figure 2). They had searched the hospital records of the era preceding adoption of metastasectomy in their hospital, seeking patients in earlier years who would have satisfied their current criteria. They found 12 . Three had lived for more than 5 years. It is a small number, and the $95 \%$ confidence limits around a $25 \%$ survival rate are wide at $5.5 \%$ to $57 \%$, but it makes zero 5 -year survival

From the ${ }^{\mathrm{a}}$ Clinical Operational Research Unit and ${ }^{\mathrm{c}}$ Surgical and Interventional Trials Unit, University College London, London, United Kingdom; ${ }^{\mathrm{b} J a m e s}$ Cook University Hospital, Middlesbrough, United Kingdom; and ${ }^{\mathrm{d}}$ Centre for Trials Research, Cardiff University, Cardiff, United Kingdom.

Received for publication July 19, 2020; revisions received Nov 19, 2020; accepted for publication Jan 2, 2021; available ahead of print March 9, 2021.

Address for reprints: Tom Treasure, MD, MS, FRCS, FRCP, Clinical Operational Research Unit, University College London, 4 Taviton St, London WC1H 0BT, United Kingdom (E-mail: tom.treasure@gmail.com).

J Thorac Cardiovasc Surg 2022;163:486-90

$0022-5223 / \$ 36.00$

Copyright (c) 2021 by The American Association for Thoracic Surgery

https://doi.org/10.1016/j.jtcvs.2021.01.142

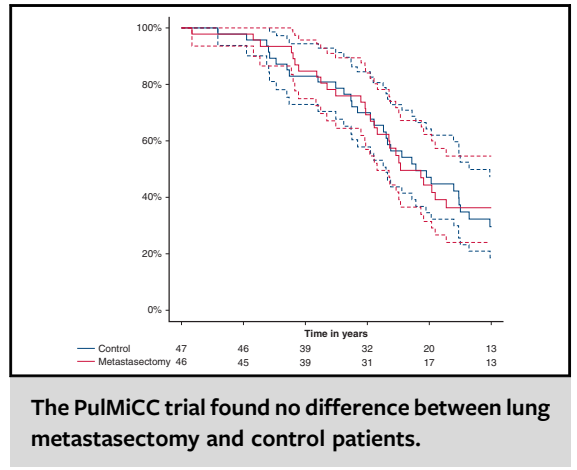

CENTRAL MESSAGE

A randomized controlled trial found no survival or quality of life benefit from lung metastasectomy; the control patients lived as long and as well as those who underwent a metastasectomy operation.

See Commentaries on pages 491 and 493 .

improbable. The article by Åberg and colleagues ${ }^{3}$ has the word metastasectomy in the title, and was published in Annals of Thoracic Surgery, but in a citation network analysis it was only cited twice among the 101 articles reviewed. ${ }^{4}$ Thereafter, uncited, the article dropped out of sight.

Of 101 articles, 51 contained data suitable for analysis on 3504 patients. $^{2}$ None included control data or estimates of survival without metastasectomy. Solitary metastases were removed in $60 \%$, more than $60 \%$ had no carcinoembryonic antigen elevation, and the interval since the primary CRC resection shortened from $\sim 3$ to 2 years during about 40 years of clinical experience. To derive an estimate of what the survival without metastasectomy might be, the Clinical Operational Research Unit worked with the Thames Cancer Registry to perform a mathematical modeling exercise. ${ }^{5} \mathrm{~Pa}-$ tients in the Registry whose age, sex, cancer stage, and deathfree survival were similar to those in large clinical series, and had a survival rate much higher than had been assumed.

The models informed the cautious power calculation of the PulMiCC trial, which was designed to show 


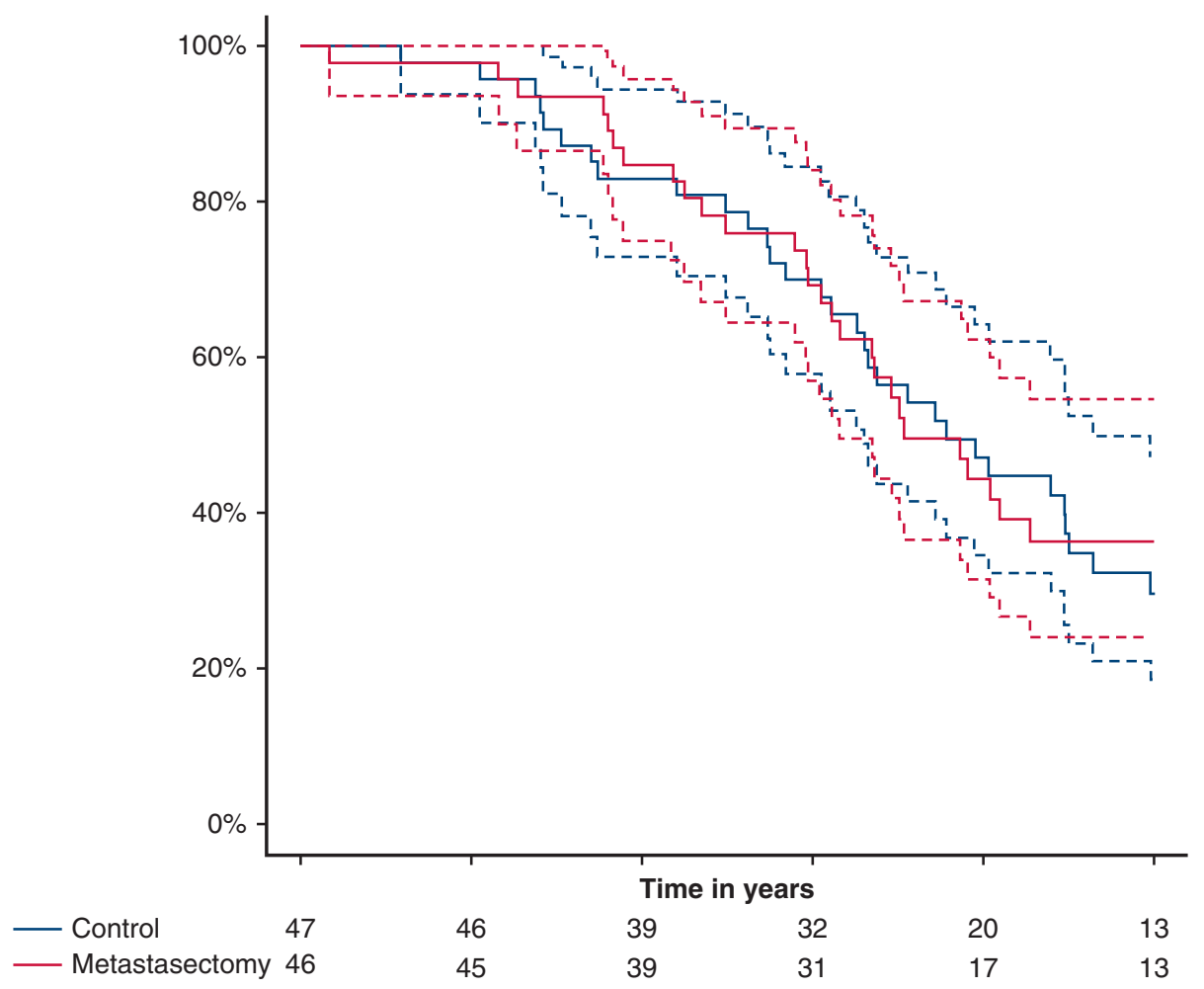

FIGURE 1. The Kaplan-Meier survival curves of the randomized controlled trial Pulmonary Metastasectomy in Colorectal Cancer (PulMiCC) in 93 patients. Overall hazard ratio, $0.93 ; 95 \%$ confidence interval, $0.56-1.56$.

noninferiority of nonmetastasectomy. PulMiCC opened in 14 centers and from December 2010 to November 2016 randomized 93 patients with CRC lung metastases, fewer than were hoped for. Of 512 patients who gave informed written consent to enter the study for evaluation, $82 \%$ were not

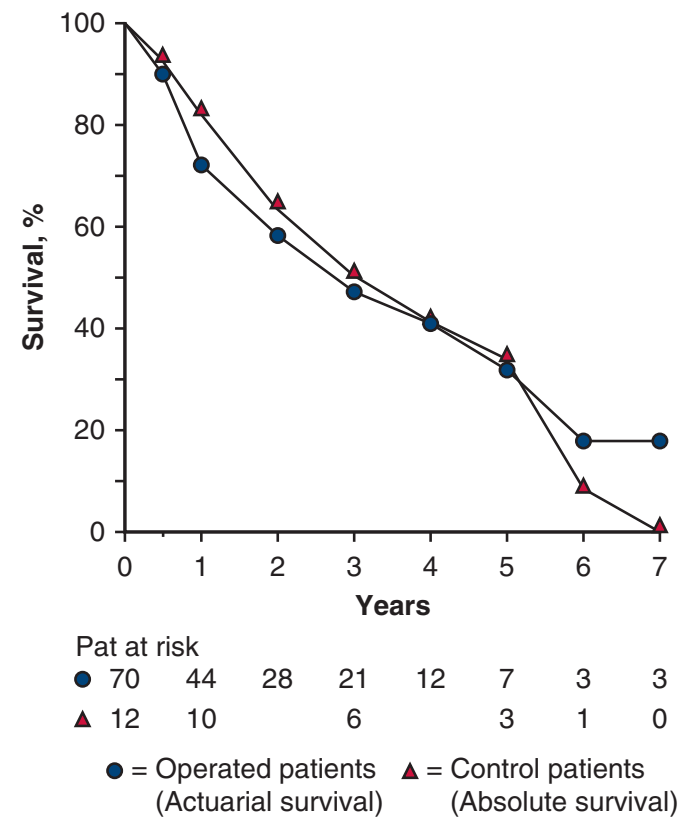

FIGURE 2. A comparative study in $1980 .^{3}$ It explicitly contradicted the assumed zero survival. Reprinted with permission. randomized. At the behest of our Data Monitoring and Ethics Committee, the reasons for failure to randomize eligible patients from the 3 largest recruiting centers was investigated. Among 155 patients, there were 41 patients who elected to make their own decision, 19-nearly half-chose not to have metastasectomy. For 78 patients, the multidisciplinary team made the decision and 77 $(99 \%)$ underwent operation. ${ }^{6}$ The patients showed equipoise, whereas the clinicians did not, probably because of the widespread conviction that without lung metastasectomy, none of these patients would survive. ${ }^{7}$ There was also pressure on them from clinical colleagues to fall in line with accepted practice in the management of metastatic colorectal cancer. ${ }^{8}$

In PulMiCC, the known confounding factors were balanced by including a minimization step in randomization. The characteristics of the patients were in line with published research (Table 1), except for the proportion of solitary metastases, which was 37\% (34 out of 93) in PulMiCC, compared with $63 \%$ in a meta-analysis, including nearly 3000 patients. ${ }^{9}$ This reflects reluctance on the part of multidisciplinary teams to randomize patients with a solitary metastasis. Multiple versus solitary metastases is associated with lower survival (hazard ratio, 2.04). ${ }^{9}$ The overall 5 -year survival of patients assigned to metastasectomy was $36 \%(95 \%$ confidence interval [CI], 15\%-46\%) in PulMiCC compared with $42 \%$ in the meta-analysis, 
TABLE 1. Characteristics used in minimization

\begin{tabular}{|c|c|c|}
\hline Characteristic & $\begin{array}{c}\text { Group 1: } \\
\text { Control }(n=47)\end{array}$ & $\begin{array}{c}\text { Group 2: } \\
\text { Metastasectomy } \\
(\mathrm{n}=46)\end{array}$ \\
\hline \multicolumn{3}{|l|}{ Sex } \\
\hline Male & 28 & 31 \\
\hline Female & 19 & 15 \\
\hline \multicolumn{3}{|l|}{ Age $(y)$} \\
\hline$\geq 61$ & 33 & 32 \\
\hline$\leq 60$ & 14 & 14 \\
\hline \multicolumn{3}{|l|}{ Lung metastases } \\
\hline 1 & 16 & 18 \\
\hline 2 to 4 & 26 & 24 \\
\hline $5+$ & 5 & 4 \\
\hline \multicolumn{3}{|l|}{ CEA (ng/mL) } \\
\hline$<5$ & 36 & 37 \\
\hline $5-10$ & 6 & 6 \\
\hline$\geq 10$ & 5 & 3 \\
\hline \multicolumn{3}{|l|}{ Prior liver resection } \\
\hline Yes & 13 & 14 \\
\hline No & 34 & 32 \\
\hline \multicolumn{3}{|c|}{ Years since $1^{\circ}$} \\
\hline \multicolumn{3}{|l|}{ CRC resection } \\
\hline$<1$ & 7 & 7 \\
\hline $1-3$ & 28 & 26 \\
\hline$\geq 3$ & 12 & 13 \\
\hline \multicolumn{3}{|l|}{ CRC stage } \\
\hline \multicolumn{3}{|l|}{$\mathrm{T}$ stage } \\
\hline 1 & 2 & 2 \\
\hline 2 & 8 & 7 \\
\hline$\geq 3$ & 37 & 37 \\
\hline \multicolumn{3}{|l|}{ N Stage } \\
\hline 0 & 25 & 24 \\
\hline$\geq 1$ & 22 & 22 \\
\hline
\end{tabular}

Data obtained at baseline in all patients and used in the minimization step in randomizing patients to the 2 trial arms. $C E A$, Carcinoembryonic antigen; $C R C$, colorectal cancer.

consistent with fewer solitary metastases being randomized. Among PulMiCC patients, 5-year survival of patients with a solitary metastasis was similar at 6 out of 16 in the control arm and 5 out of 18 in the metastasectomy arm. ${ }^{1}$ No control patients crossed over to have metastasectomy, or any form of ablation, as the initial treatment for their lung metastases. Subsequent treatments, including chemotherapy and radiotherapy, were few and similar in the 2 arms. $^{1}$

In PulMiCC, the median survival was 3.5 years in the metastasectomy arm compared with 3.8 years for control patients. It is worth noting that a 3- to 4-month difference might be regarded as worthwhile in much larger trials of chemotherapy, but it was not significant although it signaled in favor of control. Scrutiny of the survival curves shows 2 lines weaving in and out of each other (Figure 1). At 4 years, the overall estimated survival was $47 \%$ (95\% CI, 32\%$63 \%$ ) for control patients and 44\% (95\% CI, 29\%-61\%) for metastasectomy patients. Overall, the hazard ratio was
0.93 (95\% CI, 0.56-1.56). The results cannot exclude the possibility of occasional long-term survival, where metastasectomy appears to have removed the only residual disease. That would allow for the anecdotal cases that colleagues recall, but they are few and not well documented.

Other than the small expected fall in tests of lung function during the first 3 months, there were no differences in quality of life. ${ }^{6}$ The Health Utility instrument EuroQol 5 dimensions, 3 levels, showed similar losses in self-reported health status during the first 2 years after randomization (Figure 3). ${ }^{10}$

At a sample size of 93, PulMiCC is large enough to draw some important conclusions about the true effect on survival. If the zero assumption was correct, the results should have been 0 out of 47 control survival versus 17 out of $46(37 \%)$ among randomly assigned patients (Fisher exact test $P<.0001)$. However, the published estimate in the report of the meta-analysis was moderated to "worse than $5 \%$," without credible evidence. ${ }^{9}$ Running the Fisher exact test around the $5 \%$ estimate, for 2 out of $47(4 \%)$ and 3 out of $47(6 \%)$, Fisher exact test gives a $P$ value for difference $<.0001$ and $<.0003$, respectively. If such results had emerged from PulMiCC, it is unlikely the trial would have been rejected because of small numbers, irrespective of any prior power calculation. The repeated dismissal of PulMiCC as too small is surely because it was out of kilter with prior consensus. ${ }^{7}$ Power calculations are done to reconsider trial designs with no realistic prospect of answering the research question, but once the trial is done and the data are in, the power calculation becomes irrelevant in the actual data analysis. ${ }^{11}$ PulMiCC data are the most reliable available and in any future trial, the power calculation would have to take them into account in deciding the effect size to be used in determination of the sample size.

It is clear from PulMiCC that the survival of untreated patients is much higher than has been widely believed. The same has been seen in the only other 2 RCTs testing local treatment of metastases, with radiofrequency ablation and with stereotactic radiotherapy and reporting overall survival. ${ }^{12,13}$ The authors of an RCT on liver metastasectomy wrote: "The study shows that local tumor ablation by radiofrequency ablation in combination with systemic therapy results in an excellent survival, which however was also achieved in the control arm." 14 Their interpretation lacks objectivity and their findings were at odds with the assumed near-zero survival assumption. The control patients in that trial, and in PulMiCC, provide a pooled total of 106 patients, eligible for local treatment of CRC metastases in the liver or lung. There was 30\% 5-year survival; the $95 \% \mathrm{CI}$, derived using a complementary log-log scale, is $21 \%$ to $40 \%$.

The question that arises is whether the better than anticipated control results are due to improving overall survival with the newer treatments. A 2015 systematic review published in JAMA Oncology concluded: "Gains from firstline therapies have been modest but consistent; however, gains from second-line therapies have been 

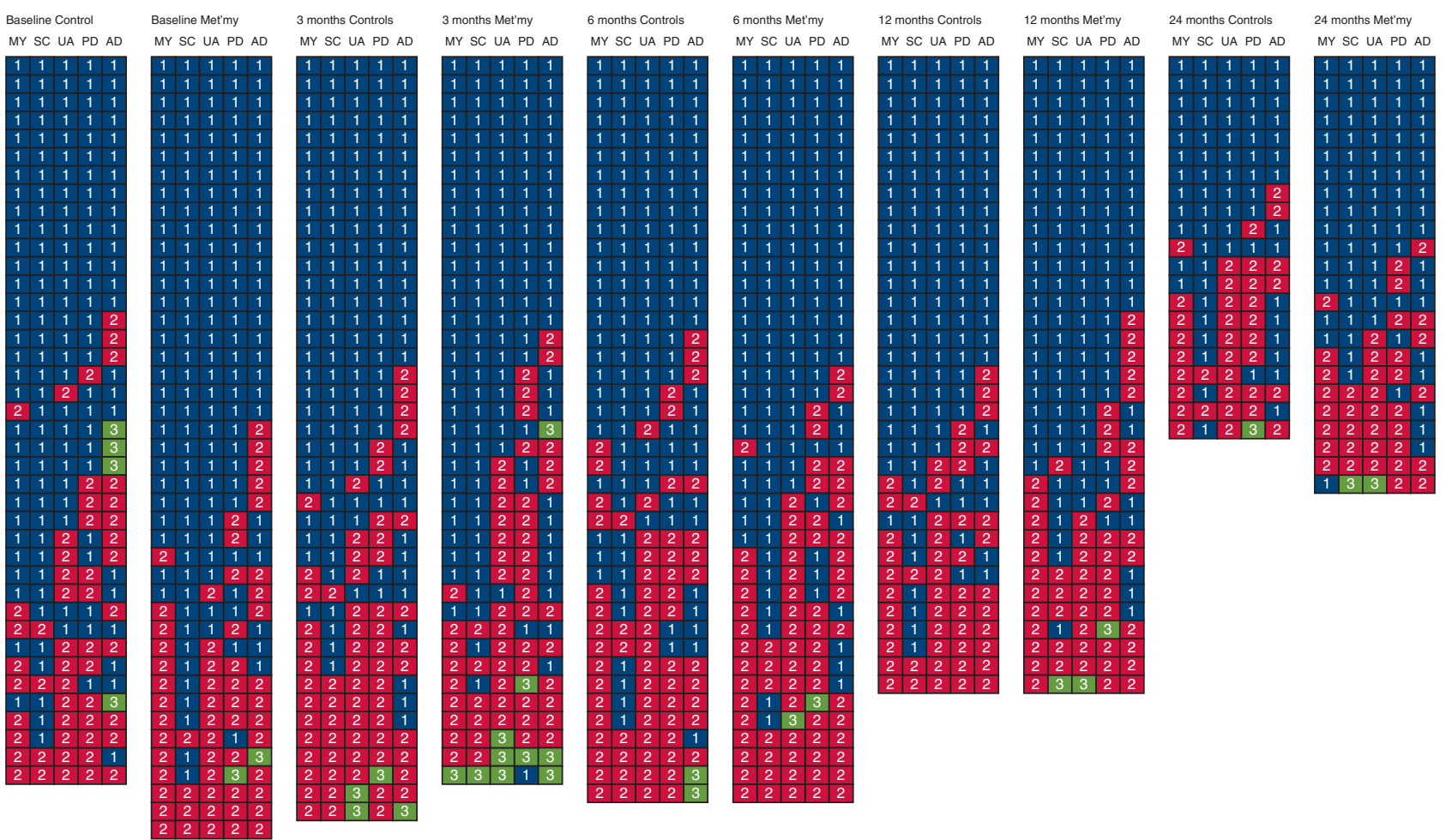

FIGURE 3. 3-Level scores in which 1 (blue) is unimpaired, 2 (red) is moderate impairment, and 3 (green) is severe impairment in the 5-Dimensions of EuroQol (EQ-5D-3L) at baseline, 3, 6, 12, and 24 months, in the control (left) and metastasectomy arms. Each horizontal set of 5 represents an individual's self-report of mobility, self-care, usual activity, pain/discomfort, and anxiety/depression sorted vertically in order of diminishing health state.

disappointing." 15 The meta-analysts pointed out the effect of lead-time bias: if diagnosis of recurrence is made sooner, it adds to time alive, creating a false impression of improved care. That said, multiple RCTs have shown that more intensive monitoring does lead to earlier diagnosis, but this has not led to beneficial survival effect. ${ }^{16,17}$ The advantage of an RCT is that any of these gains, true or illusory, apply to both arms. Furthermore, Jawed and colleagues ${ }^{15}$ flag the possibility that increasing numbers of metastasectomies may be due to the increased opportunities presented by longer survival, rather than the operations being the cause of longer survival. Critical to this discussion, they emphasize that "the most important conclusion to be drawn from this analysis is the indisputable value of enrolling patients in clinical trials." 15

The apparently universal acceptance of near-zero survival raises more general points about cognitive bias and how opinions can override facts. ${ }^{18}$ With constant repetition, falsehoods may be perceived as the truth. ${ }^{19}$ Human beings draw inferences from consistency with knowledge and may be resistant to updating beliefs when facts change. At the first public presentation of the survival graph of PulMiCC at a conference bearing the title, Preventing Overdiagnosis and Over Treatment, a thoracic surgeon rose and forthrightly declared that this RCT would convince nobody and that the control findings were erroneous because Big Data show nobody survives with unresected lung metastases. Our colleague may be correct on the first point- the psychology research cited above supports his comment that evidence may not convince people to change their opinions $^{20}$ - but on the second point, there is an important misconception. Big databases include all patients with metastatic disease, rather than the $2 \%$ to $3 \%$ selected for metastasectomy. ${ }^{21,22}$ Furthermore, Big Data misses prognostic factors-most of the known and all the unknown-so however big the collection of data, conclusions are less reliable than a careful RCT. Databases of cancer treatments record therapeutic events. They cannot provide equivalent data on identical patients who, for whatever reason, did not have the treatment. Nor do surgeons have ready access to the outcomes of patients whom they have never met. Ảberg and colleagues ${ }^{3}$ had to search for 12 comparator patients. Clinicians at the sharp end may overestimate how large a proportion of patient survival is due to their efforts, and how much due to the selection of naturally longer surviving patients. Uncontrolled observational studies of other ablative modalities are being added to the literature at an alarming rate, in the belief that they can replicate the proven benefits of surgery with less-invasive methods.

Our article dwells on lung metastases from colorectal cancer. This is because it is the largest component of lung metastasectomy practice and therefore the most amenable to clinical trial and database research. It is likely that much of what has been learned during the past 15 years applies to other carcinomas. Sarcoma has a predilection to metastasize to the lung, and affects young people. ${ }^{23}$ More often than 
not, further metastases become evident and the policy that has evolved is that in patients where the cancer runs an indolent course, reoperations are performed, selectively, until the loss of pulmonary function calls a halt. There are no controlled studies to prove that it is surgery, rather than selection for surgery, that leads to an apparent association between lung metastasectomy and survival. Germ cell tumors are treated systemically and for them, lung metastasectomy may have a place in removing a necrotic lung mass and gleaning information about tumor response.

In medicine there must be retreats as well as advances. ${ }^{24}$ Remember that radical mastectomy was the standard treatment for breast cancer for 90 years, until a trial of quite modest size displaced it. We need reliable RCTs to guide management of patients, not biased observational studies and belief.

What should be the next step? We believe that these findings should ideally be confirmed (or refuted) in a larger RCT of local treatment of metastases (surgical or radiofrequency ablation or stereotactic ablative radiotherapy) compared with no intervention, powered to show a clinically relevant improvement in 5-year survival. If this did confirm that there is a benefit, and the design included stratification with minimization, it might also indicate for which patients it is most effective. Such a trial may be difficult because of the prevailing belief in effectiveness despite the lack of evidence. But it is now essential to avoid possible wasted resources and avoidable harm to patients. Current and planned trials comparing different local treatments, and trials adding systemic therapy to one arm of a trial in which both arms undergo metastasectomy, ${ }^{25}$ cannot answer the question. Trials that have progression-free survival as the primary outcome are potentially misleading. Overall survival and health utility are the relevant outcomes. The cold light of reliable evidence still needs to be shone on this very uncertain area of oncology practice.

\section{Conflicts of Interest Statement}

The authors reported no conflicts of interest.

The Journal policy requires editors and reviewers to disclose conflicts of interest and to decline handling or reviewing manuscripts for which they may have a conflict of interest. The editors and reviewers of this article have no conflicts of interest.

\section{References}

1. Milosevic M, Edwards J, Tsang D, Dunning J, Shackcloth M, Batchelor T, et al. Pulmonary metastasectomy in colorectal cancer: updated analysis of 93 randomized patients - control survival is much better than previously assumed. Colorect Dis. 2020;22:1314-24.

2. Fiorentino F, Hunt I, Teoh K, Treasure T, Utley M. Pulmonary metastasectomy in colorectal cancer: a systematic review and quantitative synthesis. J R Soc Med. 2010;103:60-6.

3. Ảberg T, Malmberg KA, Nilsson B, Nou E. The effect of metastasectomy: fact or fiction? Ann Thorac Surg. 1980;30:378-84.

4. Fiorentino F, Vasilakis C, Treasure T. Clinical reports of pulmonary metastasectomy for colorectal cancer: a citation network analysis. Br J Cancer. 2011;104:1085-97.
5. Utley M, Treasure T, Linklater K, Moller H. Better out than in? The resection of pulmonary metastases from colorectal tumours. In: Xie X, Lorca F, Marcon E, eds. Operations Research for Health Care Engineering: Proceedings of the $33^{\text {rd }}$ International Conference on Operational Research Applied to Health Services. Saint-Etienne, France: Publications de l'Universitaire de Saint-Etienne; 2008:493-500.

6. Treasure T, Farewell V, Macbeth F, Monson K, Williams NR, Brew-Graves C, et al. Pulmonary metastasectomy versus continued active monitoring in colorectal cancer (PulMiCC): a multicentre randomised clinical trial. Trials. 2019;20:718.

7. Handy JR, Bremner RM, Crocenzi TS, Detterbeck FC, Fernando HC, Fidias PM, et al. Expert consensus document on pulmonary metastasectomy. Ann Thorac Surg. 2019;107:631-49.

8. Dawson P. ACPGBI IMPACT initiative: improving management of patients with advanced colorectal tumours: Association of Coloproctology of Great Britain and Ireland. Available at: https://www.acpgbi.org.uk/content/uploads/2017/02/ ACPGBI-Advanced-Malignancy-Initiative-15-May-2017-v4.pdf. Accessed March 15, 2021.

9. Gonzalez M, Poncet A, Combescure C, Robert J, Ris HB, Gervaz P. Risk factors for survival after lung metastasectomy in colorectal cancer patients: a systematic review and meta-analysis. Ann Surg Oncol. 2013;20:572-9.

10. Brew-Graves C, Farewell V, Monson K, Milosevic M, Williams N, Morris E, et al. Pulmonary metastasectomy in colorectal cancer: health utility scores by EQ-5D-3L in a randomised controlled trial show no benefit from lung metastasectomy. Colorect Dis. 2021;23:200-5.

11. Cox DR. Planning of Experiments. New York, NY: Wiley; 1958.

12. Ruers T, van Coevorden F, Punt CJ, Pierie JE, Borel-Rinkes I, Ledermann JA, et al. Local treatment of unresectable colorectal liver metastases: results of a randomized phase II trial. J Natl Cancer Inst. 2017;109:djx015.

13. Palma DA, Olson R, Harrow S, Gaede S, Louie AV, Haasbeek C, et al. Stereotactic ablative radiotherapy versus standard of care palliative treatment in patients with oligometastatic cancers (SABR-COMET): a randomised, phase 2, openlabel trial. Lancet. 2019;393:2051-8.

14. Ruers T, Punt C, van Coevorden F, Pierie JP, Borel-Rinkes I, Ledermann JA, et al. Radiofrequency ablation combined with systemic treatment versus systemic treatment alone in patients with non-resectable colorectal liver metastases: a randomized EORTC Intergroup phase II study (EORTC 40004). Ann Oncol. 2012;23:2619-26.

15. Jawed I, Wilkerson J, Prasad V, Duffy AG, Fojo T. Colorectal cancer survival gains and novel treatment regimens: a systematic review and analysis. JAMA Oncol. 2015;1:787-95.

16. Mokhles S, Macbeth F, Farewell V, Fiorentino F, Williams NR, Younes RN, et al. Meta-analysis of colorectal cancer follow-up after potentially curative resection. Br J Surg. 2016;103:1259-68.

17. Jeffery M, Hickey BE, Hider PN. See AM. Follow-up strategies for patients treated for non-metastatic colorectal cancer. Cochrane Database Syst Rev. 2016;11:CD002200.

18. Lewandowsky S, Ecker U, Cook J. Beyond misinformation: understanding and coping with the "post-truth" era. J Appl Res Mem Cognit. 2020;6:353-69.

19. Dechene A, Stahl C, Hansen J, Wanke M. The truth about the truth: a metaanalytic review of the truth effect. Pers Soc Psychol Rev. 2010;14:238-57.

20. Macbeth F, Fallowfield L. The myth of pulmonary metastasectomy. Br J Cancer. 2020;123:499-500.

21. Embun R, Fiorentino F, Treasure T, Rivas JJ, Molins L. Pulmonary metastasectomy in colorectal cancer: a prospective study of demography and clinical characteristics of 543 patients in the Spanish colorectal metastasectomy registry (GECMP-CCR). BMJ Open. 2013;3:e02787.

22. Fenton H, Finan PJ, Milton R, Shackcloth M, Taylor J, Treasure T, et al. National variation in pulmonary metastasectomy for colorectal cancer. Colorectal Dis. 2021;23:1306-16.

23. Treasure T, Fiorentino F, Scarci M, Moller H, Utley M. Pulmonary metastasectomy for sarcoma: a systematic review of reported outcomes in the context of Thames Cancer Registry data. BMJ Open. 2012;2:e00736.

24. Prasad V, Cifu A. Ending Medical Reversal: Improving Outcomes, Saving Lives. Baltimore, MD: Johns Hopkins University Press; 2015.

25. Corsini EM, Mitchell KG, Correa A, Morris VK, Antonoff MB, MD Anderson Pulmonary Metastasectomy Working Group. Effect of primary colorectal cancer tumor location on survival after pulmonary metastasectomy. J Thorac Cardiovasc Surg. 2021;162:296-305.

Key Words: metastasectomy, colorectal cancer 